\title{
Improving Amplify-and-Forward Relay Networks: Optimal Power Allocation versus Selection
}

\author{
Yi Zhao, Raviraj Adve and Teng Joon Lim \\ Dept. of Electrical and Computer Engineering, \\ University of Toronto, \\ Toronto, ON, M5S3G4, Canada. \\ E-mail: zhaoyi, rsadve, limtj@comm.utoronto.ca.
}

\begin{abstract}
We consider an Amplify-and-Forward cooperative diversity system where a source node communicates with a destination node with the help of one or more relay nodes. The conventional system model assumes all relay nodes participate, with the available channel and power resources equally distributed over all nodes. This approach being clearly sub-optimal, we first present two power allocation schemes to minimize the system outage probability, based on complete channel state information and channel statistics, respectively. We further show that the proposed optimal power allocation methods minimize system symbol error rate as well. Next, we propose a selection scheme where only one "best" relay node is chosen to assist in the transmission. We show that the selection-AF scheme maintains full diversity order, and at reasonable power levels has significantly better outage behavior and average throughput than the conventional all-participate scheme or that with optimal power allocation. Finally we combine power allocation and selection to further improve performance.
\end{abstract}

Keywords: Cooperative System, Amplify-and-Forward, Optimal Power Allocation, Selection Scheme.

\section{INTRODUCTION}

Prior work has shown that a cooperative diversity gain is available in distributed wireless networks where nodes help each other by relaying transmissions [1]-[3]. The system under consideration comprises a source, a destination and $m$ potential relay nodes. The most popular cooperation protocols are amplifyand-forward ( $\mathrm{AF}$ - the relay simply amplifies the source transmission and retransmits) and decode-andforward (DF - the relay decodes the source transmission, re-encodes and retransmits). Laneman and Wornell [1] studied an AF scheme in which all $m$ relays help the source, $(m+1)$ orthogonal channels are used for transmission, and the total available power is equally divided up among the $(m+1)$ transmitting nodes. This scheme achieves order- $(m+1)$ diversity. Clearly, the performance of such an "all participate" amplify-and-forward (AP-AF) network with equal power allocation (EPA) is upper-bounded by the case of using perfect knowledge of all channel gains for optimal power allocation (OPA). 
OPA in AF networks has been studied recently in [4]-[11]. Most of these (e.g., [4]-[9]) focus on the single-relay case, and solve for the optimal power division between the source and relay nodes to maximize capacity [4], [5], minimize transmission power [6], minimize outage probability [7], [8] or probability of error [9]. However, the extension of these algorithms to multiple relays is not obvious. OPA in multi-hop systems was discussed in [10], where the relay nodes are used to extend the coverage area, rather than to provide diversity for improving throughput or reducing outage probability. Employing multiple relay nodes with distributed beamforming for diversity gain was studied in [11].

This paper first revisits Laneman's framework in [1], where system resources are shared and nodes transmit in orthogonal channels. We derive two OPA algorithms for AP-AF networks with multiple relay nodes to minimize outage probability, with two different levels of channel knowledge: complete channel state information (CSI) and channel statistics. The OPA schemes are presented as extended water-filling processes under both total and individual power constraints. Furthermore, although our goal of power allocation is to maximize the throughput or minimize the outage probability, we prove that these OPA schemes also minimize symbol error rate (SER). These power allocation schemes are different from those in a conventional multi-input multi-output (MIMO) system since in a cooperative network, every relay has its own orthogonal channel, while multiple antennas transmit and interfere in the same channel.

The second half of the paper recognizes the fact that despite optimal power allocation, the performance of the AP-AF scheme is fundamentally limited by the orthogonal partitioning of system resources. This overhead to cooperation is particularly damaging in large networks with many nodes. To solve this problem, this paper introduces a selection-based cooperation structure, selection amplify-and-forward (S-AF), in which only one "best" node is chosen as a relay. A selection scheme over cooperative networks has been introduced by Bletsas et al. [12]. In their work the selection is based on a delay process at the relays, and the selection criterion is not optimal. Furthermore, their selection process may fail due to packet collision and the analysis in [12] quantifies the probability of collisions. In [13] a selection DF scheme is introduced, however, the underlying cooperation mechanism and partner selection algorithms are very different. Our selection algorithm, building on the work in [13], is implemented at the destination, which is assumed to have knowledge of all channel gains, including those between the source and all relays, hence the selection algorithm can be designed to minimize the outage probability directly.

As we will show, both S-AF and AP-AF achieve the maximum diversity order of $(m+1)$. But more importantly, at reasonable power levels, we show that whenever more than two relays are available $(m>2)$, 
S-AF achieves a higher instantaneous throughput, and hence lower outage probability than AP-AF. These analytical results can be justified intuitively: (a) in a distributed network instead of distributing the channel resources over $(m+1)$ nodes for transmitting information, S-AF only halves the resources, and (b) since S-AF chooses the best of $m$ relays, the relays still provide a diversity order of $m$. At the end of this paper we combine OPA and relay selection ${ }^{1}$ for better performance. Simulation results show that this combined selection with OPA scheme achieves best performance among all the schemes in this paper.

The rest of the article is organized as follows. Section II introduces the conventional AF system model, and analyzes its outage behavior. Section III sets up the optimal power allocation problems for the AP-AF scheme and presents solutions for two special cases of practical interest: with complete CSI and with only channel statistics. Section IV presents the S-AF scheme and compares it with AP-AF for both throughput and outage probability. Finally, Section V concludes the paper.

\section{All-PARTicipate AMPLIFY-AND-ForWARD}

Consider a system in which a source node ' $s$ ' transmits information to a destination node ' $d$ ' with the help of $m$ relay nodes. Transmissions use orthogonal channels, either through time or frequency division. For convenience, we assume time division and so each node is assigned one of $(m+1)$ time slots in each information packet.

\section{A. System Model}

In the first, data-sharing, time slot, the source node transmits to the destination as well as the relay nodes. The signals received at the destination and the relays are

$$
\begin{aligned}
y_{s, d} & =\sqrt{E_{s}} h_{s, d} x+n_{s, d}, \\
y_{s, i} & =\sqrt{E_{s}} h_{s, i} x+n_{s, i}, \quad i=1, \cdots, m,
\end{aligned}
$$

where $x, y_{s, d}$ and $y_{s, i}$ denote the (unit energy) transmitted signal and the signals received at the destination and the $i$ th relay node, respectively. $h_{s, i}$ and $h_{s, d}$ are channel coefficients of the source-relay and sourcedestination channels, which include the effect of shadowing, channel loss and fading. $E_{s}$ is the average energy transmitted in this time slot. Assuming all the time slots have unit duration, $E_{s}$ can be considered as the transmission power. $n_{s, d}$ and $n_{s, i}$ are additive circularly symmetric white Gaussian noise (AWGN)

\footnotetext{
${ }^{1}$ By solving the problem of allocating power optimally between source and selected relay.
} 
in the corresponding channels, with variance $N_{s, d}$ and $N_{s, i}$, respectively, i.e., $n_{s, d} \sim \mathcal{C N}\left(0, N_{s, d}\right), n_{s, i} \sim$ $\mathcal{C N}\left(0, N_{s, i}\right)$.

In $m$ subsequent time slots, the $m$ relays use the AF protocol, i.e., normalize their received signals and retransmit to the destination one at a time. For the $i$ th relay, the normalization factor is $\sqrt{\mathrm{E}\left\{\left|y_{s, i}\right|^{2}\right\}}$ (where $\mathrm{E}\{\cdot\}$ denotes the expectation operator) and thus the signal transmitted from the $i^{\text {th }}$ relay is

$$
x_{i}=\frac{y_{s, i}}{\sqrt{\mathrm{E}\left\{\left|y_{s, i}\right|^{2}\right\}}}=\frac{\sqrt{E_{s}} h_{s, i} x+n_{s, i}}{\sqrt{E_{s}\left|h_{s, i}\right|^{2}+N_{s, i}}} .
$$

Note that we assume that $E_{s},\left|h_{s, i}\right|$ and $N_{s, i}$ are known at the $i$ th relay.

Based on (3), the signal received by the destination from the $i$ th relay node is

$$
y_{i, d}=\sqrt{E_{i}} h_{i, d} x_{i}+n_{i, d}=\sqrt{\frac{E_{s} E_{i}}{E_{s}\left|h_{s, i}\right|^{2}+N_{s, i}}} h_{i, d} h_{s, i} x+\tilde{n}_{i, d},
$$

where $h_{i, d}$ is the channel gain from node $i$ to the destination, and $E_{i}$ is the power used by node $i$ for transmission in its time slot. $n_{i, d} \sim \mathcal{C N}\left(0, N_{i, d}\right)$ denotes the AWGN of the relay-destination channel. $\tilde{n}_{i, d}$ is the equivalent noise term in $y_{i, d}$. It can be easily shown that $\tilde{n}_{i, d} \sim \mathcal{C N}\left(0, \tilde{N}_{i, d}\right)$ with

$$
\tilde{N}_{i, d}=N_{i, d}+\frac{E_{i}\left|h_{i, d}\right|^{2} N_{s, i}}{E_{s}\left|h_{s, i}\right|^{2}+N_{s, i}}
$$

\section{B. Outage Performance and Diversity Analysis}

We can write the normalized received signals from all the time slots in vector form as [14]:

$$
\mathbf{y}_{d}=\mathbf{h} x+\mathbf{n}
$$

where

$$
\begin{gathered}
\mathbf{y}_{d}=\left[\begin{array}{lllll}
\frac{y_{s, d}}{\sqrt{N_{s, d}}} \frac{y_{1, d}}{\sqrt{\tilde{N}_{1, d}}} & \cdots & \frac{y_{i, d}}{\sqrt{\tilde{N}_{i, d}}} & \cdots & \frac{y_{m, d}}{\sqrt{\tilde{N}_{m, d}}}
\end{array}\right]^{T} \\
\mathbf{h}=\left[\begin{array}{llll}
\frac{\sqrt{E_{s}} h_{s, d}}{N_{s, d}} & \frac{1}{\sqrt{\tilde{N}_{1, d}}} \sqrt{\frac{E_{s} E_{1}}{E_{s}\left|h_{s, 1}\right|^{2}+N_{s, 1}}} h_{1, d} h_{s, 1} & \cdots & \frac{1}{\sqrt{\tilde{N}_{m, d}}} \sqrt{\frac{E_{s} E_{m}}{E_{s}\left|h_{s, m}\right|^{2}+N_{s, m}}} h_{m, d} h_{s, m}
\end{array}\right]^{T}
\end{gathered}
$$

with $\mathbf{n} \sim \mathcal{C N}(\mathbf{0}, \mathbf{I})$ and $\tilde{N}_{i, d}$ defined in (5). Note that normalizing the received signal $y_{i, d}$ with $\tilde{N}_{i, d}$ does not change the signal-to-noise ratio (SNR), but the resulting identity noise covariance matrix simplifies the computations needed later. Thus the source-destination channel capacity, for a given $\mathbf{h}$, when all nodes 
participate is

$$
\begin{aligned}
\mathcal{I}_{A P} & =\frac{1}{m+1} \log _{2}\left(1+\mathbf{h}^{H} \mathbf{h}\right) \\
& =\frac{1}{m+1} \log _{2}\left[1+\frac{E_{s}\left|h_{s, d}\right|^{2}}{N_{s, d}}+\sum_{i=1}^{m} \frac{\frac{E_{s}\left|h_{s, i}\right|^{2}}{N_{s, i}} \frac{E_{i}\left|h_{i, d}\right|^{2}}{N_{i, d}}}{\frac{E_{s}\left|h_{s, i}\right|^{2}}{N_{s, i}}+\frac{E_{i}\left|h_{i, d}\right|^{2}}{N_{i, d}}+1}\right],
\end{aligned}
$$

in bits per time slot.

Let $N_{s, d}=N_{0}, N_{s, i}=\eta_{s, i} N_{0}$ and $N_{i, d}=\eta_{i, d} N_{0}$, where $\eta_{s, i}$ and $\eta_{i, d}$ are determined by the ratios of $N_{s, i}$ and $N_{i, d}$ to $N_{s, d}$. Denote $E_{s}\left|h_{s, d}\right|^{2}=\alpha_{0}, E_{s}\left|h_{s, i}\right|^{2} / \eta_{s, i}=\alpha_{i}$ and $E_{i}\left|h_{i, d}\right|^{2} / \eta_{i, d}=\beta_{i}$. Assuming all the channels are Rayleigh distributed, $\alpha_{0}, \alpha_{i}$ and $\beta_{i}$ are exponentially distributed random variables. Denoting $1 / N_{0}=\gamma$, the capacity formula (9) becomes

$$
\mathcal{I}_{A P}=\frac{1}{m+1} \log _{2}\left[1+\gamma \alpha_{0}+\sum_{i=1}^{m} \frac{\gamma^{2} \alpha_{i} \beta_{i}}{\gamma \alpha_{i}+\gamma \beta_{i}+1}\right]
$$

For a given set of energy allocations $\left\{E_{s}, E_{i}\right\}_{i=1}^{m}$, channels $h_{s, d},\left\{h_{s, i}\right\}_{i=1}^{m},\left\{h_{i, d}\right\}_{i=1}^{m}$ and noise coefficients $\left\{\eta_{s, i}, \eta_{i, d}\right\}_{i=1}^{m}$, the transmitted SNR at each node, and the received SNR at the destination node are all proportional to $\gamma=1 / N_{0}$. Hence $\gamma$ serves as a measure of the system SNR.

An outage event occurs when the capacity $\mathcal{I}$ falls under a required rate $R$, with outage probability $P_{\text {out }}=P[\mathcal{I}<R]$. It is extremely difficult to directly compute the exact outage probabilities for the AP-AF schemes for arbitrary SNR because the probability density function (PDF) of $\mathcal{I}_{A P}$ is hard to obtain. However, the following theorem presents upper and lower bounds on the outage probabilities for the AP-AF scheme in the high SNR regime. These bounds also provide the achieved diversity order of the AP-AF scheme.

Theorem 1: In the high SNR regime, the outage probability of AP-AF scheme can be bounded as

$$
\frac{\lambda_{0} \Pi_{i=1}^{m}\left(\lambda_{i}+\xi_{i}\right)}{(m+1) m^{m}}\left(\frac{2^{(m+1) R}-1}{\gamma}\right)^{m+1} \leq P_{\text {out }}^{A P} \leq \frac{\lambda_{0} \Pi_{i=1}^{m}\left(\lambda_{i}+\xi_{i}\right)}{m+1}\left(\frac{2^{(m+1) R}-1}{\gamma}\right)^{m+1}
$$

with $\lambda_{0}, \lambda_{i}$ and $\xi_{i}$ the exponential distribution parameters of the Rayleigh channel amplitude squares $\alpha_{0}$, $\alpha_{i}$ and $\beta_{i}$ in (10), respectively.

Proof: See Appendix A.

Since both the upper and the lower bounds in Theorem 1 are proportional to $(1 / \gamma)^{m+1}$, as expected, the AP-AF scheme achieves full diversity order of $(m+1)$. 


\section{Optimal Power Allocation}

\section{A. Optimal Power Allocation with CSI}

AP-AF requires that complete CSI i.e., $h_{s, d}, h_{s, i}$ and $h_{i, d}$, be available at the destination node to coherently decode the signal. It is feasible then for the destination to use this information to assign transmit powers to the relay nodes. The manner in which the destination obtains the CSI is beyond the scope of this paper.

This section develops the optimal power allocation among source and relay nodes to maximize $\mathcal{I}_{A P}$ and thereby minimize outage probability. This optimization problem is modeled with both sum and individual power constraints. The $i$ th node has a power budget of $E_{i}^{\max }$ while total power available is limited to $E_{T}$, or

$$
E_{s}+\sum_{i=1}^{m} E_{i} \leq E_{T}, \quad E_{s} \leq E_{s}^{\text {max }}, \quad E_{i} \leq E_{i}^{\max }
$$

To simplify (9) denote $a_{0}=\left|h_{s, d}\right|^{2} / N_{s, d}, a_{i}=\left|h_{s, i}\right|^{2} / N_{s, i}$ and $b_{i}=\left|h_{i, d}\right|^{2} / N_{i, d}$, and we have

$$
\begin{aligned}
\mathcal{I}_{A P} & =\frac{1}{m+1} \log _{2}\left[1+E_{s} a_{0}+\sum_{i=1}^{m} \frac{E_{s} E_{i} a_{i} b_{i}}{E_{s} a_{i}+E_{i} b_{i}+1}\right] \\
& =\frac{1}{m+1} \log _{2}\left[1+E_{s}\left(\sum_{i=0}^{m} a_{i}\right)-\sum_{i=1}^{m} \frac{E_{s}^{2} a_{i}^{2}+E_{s} a_{i}}{E_{s} a_{i}+E_{i} b_{i}+1}\right] .
\end{aligned}
$$

Since $\log _{2}(1+x)$ is a strictly increasing function of $x$, based on (12) and (13), we have:

$$
\left[\begin{array}{llll}
E_{s} & E_{1} & \cdots & E_{m}
\end{array}\right]_{o p t}=\arg \underset{\substack{E_{s}+\sum_{i=1}^{m} E_{i} \leq E_{T} \\
0 \leq E_{s} \leq E_{s}^{\max }, 0 \leq E_{i} \leq E_{i}^{\max }}}{\max } E_{s}\left(\sum_{i=0}^{m} a_{i}\right)-\sum_{i=1}^{m} \frac{E_{s}^{2} a_{i}^{2}+E_{s} a_{i}}{E_{s} a_{i}+E_{i} b_{i}+1} .
$$

Solving optimization problem (14) in closed form appears to be difficult. But if we relax the problem to one with a fixed pre-determined $E_{s}$, then the new problem

$$
\left[\begin{array}{lll}
E_{1} & \cdots & E_{m}
\end{array}\right]^{o p t}=\arg \min _{\substack{\sum_{i=1}^{m} E_{i} \leq E_{r} \\
0 \leq E_{i} \leq E_{i}^{m a x}}} \sum_{i=1}^{m} \frac{E_{s}^{2} a_{i}^{2}+E_{s} a_{i}}{E_{s} a_{i}+E_{i} b_{i}+1},
$$

where $E_{r}=E_{T}-E_{s}$ is the total power constraint for the relay nodes, has a closed-form solution. The relaxed problem (15) is equivalent to having the source node transmit at some reasonable power, and then optimally allocating the remaining power among the relay nodes. Without individual power constraints, the problem can be shown (using the Lagrange multiplier method, as shown in Appendix B) to have a 
water-filling solution as

$$
E_{i}=\left(\sqrt{\frac{E_{s}^{2} a_{i}^{2}+E_{s} a_{i}}{b_{i}}} \lambda-\frac{E_{s} a_{i}+1}{b_{i}}\right)^{+}
$$

where $\lambda$ is a constant to meet the total power constraint.

Now consider the individual constraints. Since the objective function (15) is a monotonically decreasing and convex function of $E_{i}$, the optimal point must be on the boundary. This can also be easily verified by the Karush-Kuhn-Tucker (KKT) conditions [15], and hence we have the following theorem.

Theorem 2: The optimal power allocation among the relay nodes to maximize the capacity (13) of AP-AF systems, given a fixed transmit power $E_{s}$ for the source node, with total and individual power constraints, is

$$
E_{i}=\left(\sqrt{\frac{E_{s}^{2} a_{i}^{2}+E_{s} a_{i}}{b_{i}}} \lambda-\frac{E_{s} a_{i}+1}{b_{i}}\right)_{0}^{E_{i}^{\max }}
$$

where $\lambda$ is a constant chosen to satisfy the total power constraint and $(x)_{l}^{u}=l(x<l),=x(l \leq x \leq$ $u), u(x>u)$. This solution can be considered as an extended water-filling process, with each vessel having both a bottom and a lid.

In the high SNR regime, the optimal power allocation reduces to

$$
E_{i}=\left(\frac{E_{s} a_{i}}{\sqrt{b_{i}}} \lambda-\frac{E_{s} a_{i}}{b_{i}}\right)_{0}^{E_{i}^{\max }} .
$$

Finally, the destination node will notify each relay node of its assigned transmission power. If a node is assigned zero power, it remains idle in its slot. Adapting the number of slots dedicated to relaying according to channel conditions would involve an adaptive transmission structure, extra feedback and centralized control and is beyond the scope of this paper. Since instantaneous throughput is maximized for each set of channel realizations, minimum outage probability is achieved. Note that the resulting optimal power allocation scheme is significantly different from waterfilling in a conventional MIMO system both due to the presence of individual power constraints and the orthogonality of the $m$ relay transmissions.

\section{B. Optimal Power Allocation with Channel Statistics}

Section III-A presented the optimal power allocation to minimize outage probability. It requires the destination node to compute the optimal power of each relay node for every channel realization, and to notify the relay nodes of their transmission power using a feedback channel. In practical systems this 
optimal power allocation scheme may not be feasible: for instance, the feedback channel from destination to relay nodes may have very low capacity, therefore real-time power control is impossible; it is also possible that power allocation is done at the relays and accurate instantaneous channel state information (from relays to destination) is not available. In those systems, however, channel statistics may be available. We now present a new power allocation scheme based on statistical channel information.

Theorem 1 introduced upper and lower bounds on the outage probability of AP-AF scheme. Given statistical information only, we minimize the outage probability by minimizing the bounds simultaneously. Ignoring the terms independent of transmission power, we have

$$
\left[\begin{array}{llll}
E_{s} & E_{1} & \cdots & E_{m}
\end{array}\right]_{\text {opt }}=\arg \underset{\substack{E_{s}+\sum_{i=1}^{m} E_{i} \leq E_{T} \\
0 \leq E_{s} \leq E_{s}^{\max }, 0 \leq E_{i} \leq E_{i}^{\max }}}{\operatorname{man}} \lambda_{0} \prod_{i=1}^{m}\left(\lambda_{i}+\xi_{i}\right) .
$$

Since $\lambda_{0}$ is defined as the exponential parameter of $E_{s}\left|h_{s, d}\right|^{2}$, we rewrite it as

$$
\lambda_{0}=\frac{1}{\mathrm{E}\left\{E_{s}\left|h_{s, d}\right|^{2}\right\}}=\frac{1}{E_{s} \nu_{s, d}},
$$

where $\nu_{s, d}$ is the variance of the Rayleigh fading channel $h_{s, d}$, or $h_{s, d} \sim \mathcal{C N}\left(0, \nu_{s, d}\right)$. Similarly we can rewrite $\lambda_{i}$ and $\xi_{i}$ as

$$
\begin{aligned}
\lambda_{i} & =\frac{\eta_{s, i}}{E_{s} \nu_{s, i}}, \\
\xi_{i} & =\frac{\eta_{i, d}}{E_{i} \nu_{i, d}},
\end{aligned}
$$

where $h_{s, i} \sim \mathcal{C N}\left(0, \nu_{s, i}\right)$ and $h_{i, d} \sim \mathcal{C N}\left(0, \nu_{i, d}\right)$.

Again we use a pre-determined value of $E_{s}$ to simplify the optimization problem. Substituting (20) and (21) into (19), we have

$$
\left[\begin{array}{lll}
E_{1} & \cdots & E_{m}
\end{array}\right]_{o p t}=\arg \min _{\substack{\sum_{i=1}^{m} E_{i} \leq E_{r} \\
0 \leq E_{i} \leq E_{i}^{\max }}} \prod_{i=1}^{m}\left(\frac{\eta_{s, i}}{E_{s} \nu_{s, i}}+\frac{\eta_{i, d}}{E_{i} \nu_{i, d}}\right) .
$$

The solution to this minimization problem is summarized in the next theorem.

Theorem 3: The optimal power allocation for minimizing system outage probability with only channel statistics is

$$
E_{i}=\left(\frac{\sqrt{4 c_{i} E_{s} \lambda+E_{s}^{2}}-E_{s}}{2 c_{i}}\right)_{0}^{E_{i}^{\max }},
$$

where $c_{i}=\frac{\nu_{i, d} \eta_{s, i}}{\nu_{s, i} \eta_{i, d}}$ is a parameter of the channels related to relay node $i$, while $\lambda$ is chosen to satisfy the total power constraint. 
Proof: See Appendix C.

\section{Minimization of SER}

In Sections III-A and III-B the goal of power allocation was to minimize the outage probability of the AP-AF relay system. It has been proved in Lemma 5 of [16] that error probability is lower bounded by the outage probability, which implies that minimizing the outage probability may be also valid for minimizing SER. In this section, we argue that the algorithms in Theorems 2 and 3 also minimize SER for any memoryless data modulation format.

Although SER of a system is related to the modulation scheme the system adopts, it is always a monotonically increasing function of the received SNR $\gamma_{r}$, e.g., $\mathcal{Q}\left(\sqrt{2 \gamma_{r}}\right)$ for binary phase shift keying (BPSK) and $2 \mathcal{Q}\left(\sqrt{2 \gamma_{r}}\right)-\mathcal{Q}^{2}\left(\sqrt{2 \gamma_{r}}\right)$ for quadrature PSK (QPSK), where $\mathcal{Q}(x)$ is the Gaussian Q-function. As a result, when complete CSI is available for power allocation, maximizing the received SNR is the equivalent to minimizing SER.

Assuming the receiver uses maximum ratio combining (MRC), the received SNR is the sum of SNR's from the different data routes, i.e., from the direct link between source and destination nodes and from all the other relays. From system equation (6) we get

$$
\gamma_{r}=E_{s} a_{0}+\sum_{i=1}^{m} \frac{E_{s} E_{i} a_{i} b_{i}}{E_{s} a_{i}+E_{i} b_{i}+1} .
$$

Note that this is the same objective function as in minimizing outage probability (14), thus the OPA in (17) not only minimizes the outage probability of the AF system, but also minimizes the SER.

Furthermore, the asymptotic averaged SEP formula for general AF systems was recently introduced in [17] as

$$
\overline{P_{e}} \approx \frac{C(m, k)}{\gamma^{m+1}} \lambda_{0} \prod_{i=1}^{m}\left(\lambda_{i}+\xi_{i}\right),
$$

where the constant $k$ depends on modulation (e.g. $k=2$ for PSK), and $C(m, k)$ is a constant depending on the number of relay nodes $m$ and $k$. In [17], the available transmission power was divided equally among all the source and relay nodes, hence $\lambda_{0}, \lambda_{i}$ and $\xi_{i}$ are determined only by their corresponding channels. However, different transmit powers can be easily taken into account by using (20) and (21), and the SER formula becomes

$$
\overline{P_{e}} \approx \frac{C(m, k)}{\gamma^{m+1}} \frac{1}{E_{s} \nu_{s, d}} \prod_{i=1}^{m}\left(\frac{\eta_{s, i}}{E_{s} \nu_{s, i}}+\frac{\eta_{i, d}}{E_{i} \nu_{i, d}}\right)
$$


Note that the terms related to transmission power are exactly the same as in (22) to minimize outage probability. Therefore, when only channel statistics are available, the optimization problem (22) is valid for minimizing SER as well.

Thus we have shown that the power allocation algorithms in Theorems 2 and 3 minimize both the system outage probability and $\mathrm{SER}^{2}$ with any linear modulation format.

\section{Simulation Results}

This section presents results of simulations to evaluate the impact of power allocation on AP-AF networks. We simulate an AP-AF network with a source, a destination and three relay nodes $(m=3)$. Four transmission schemes are compared: OPA-CSI AP-AF is our proposed optimal scheme with complete CSI, OPA-STA is the power allocation scheme with only channel statistics, equal power allocation (EPA) $\mathrm{AP}-\mathrm{AF}$ is the conventional AF scheme where all relays use the same power $\left(E_{i}=E_{s}=E_{T} /(m+1)\right)$, and Direct Transmission is when the source transmits information to the destination directly without help from the relays. The power constraints are set at $E_{s}=1, E_{r}=m$ and $E_{i}^{\max }=2$. The variance of the Rayleigh fading channels are assumed to be determined by the locations of nodes.

This example places all nodes in a circle centered at the origin of the $x-y$ plane with radius $r=1$. The source and destination nodes are located at $(-0.5,0)$ and $(0.5,0)$, respectively. Three relay nodes are uniformly distributed in the circle. The channel between two nodes is $h_{i, j} \sim \mathcal{C N}\left(0,1 / d^{\nu}\right)$, where $d$ is the distance between the two nodes, and $\nu=2.5$ is the distance attenuation factor. For all the simulations in this article we assume that all the noise variances are equal, i.e. $\eta_{s, i}=\eta_{i, d}=1$.

[Figure 1 about here.]

Figure 1 compares the average throughput of the three schemes, where results are obtained by averaging over 5, 000 channel realizations. From the figure we can see that the OPA-CSI scheme improves the average throughput by about $2 \mathrm{~dB}$ at low $\mathrm{SNR}^{3}$. Figure 2 shows the outage probability of the four schemes. The OPA-CSI results in a gain of $1.5 \mathrm{~dB}$, but the improvement by OPA-STA is small. Note that, from these figures, as expected, direct transmission has greater average throughput, but far poorer outage probability (diversity order of 1 , not $(m+1)$ ).

[Figure 2 about here.]

\footnotetext{
${ }^{2}$ Instantaneous for Theorem 2, and averaged over the fading processes for Theorem 3.

${ }^{3} \mathrm{SNR}$ is defined as $\gamma=1 / N_{0}$.
} 


\section{SELECTION AMPLIFY-AND-ForWARD SCHEME}

\section{A. Algorithm Description}

In the previous section we showed that power allocation can improve system throughput for the AP-AF scheme. However, in order to realize orthogonal transmissions, every node can only transmit in a slot with duration $1 /(m+1)$ of the entire block. Although this orthogonal transmission can achieve full diversity order, the TDMA factor of $1 /(m+1)$ in (9) has a large adverse effect on throughput when $m$ is large.

To solve this problem, we introduce a new scheme called Selection Amplify-and-Forward (S-AF) where the transmission is divided into only two slots. The first slot implements the data-sharing phase of APAF. However, the relaying phase of S-AF contains only one slot, in which a relay node selected by the destination amplifies and forwards its received signal from the source. To focus on the idea of relay selection, we first assume equal power allocation between the source and relay nodes, i.e., $E_{s}=E_{i}$.

Using the same notation as in (10), the capacity of the source-destination channel when relay $i$ is chosen for relaying is

$$
\mathcal{I}_{S}(i)=\frac{1}{2} \log _{2}\left(1+\gamma \alpha_{0}+\frac{\gamma^{2} \alpha_{i} \beta_{i}}{\gamma \alpha_{i}+\gamma \beta_{i}+1}\right)
$$

bits per time slot. The maximum capacity is therefore attained when the relay with the largest

$$
P_{i}=\frac{\gamma^{2} \alpha_{i} \beta_{i}}{\gamma \alpha_{i}+\gamma \beta_{i}+1}
$$

is selected, resulting in a capacity of

$$
\mathcal{I}_{S}=\frac{1}{2} \log _{2}\left[1+\gamma \alpha_{0}+\max _{i} \frac{\gamma^{2} \alpha_{i} \beta_{i}}{\gamma \alpha_{i}+\gamma \beta_{i}+1}\right]
$$

The destination node needs only to select and notify the best relay, instead of computing and feeding back the power allocated to every relay node. Therefore the complexity of S-AF is lower than that of AP-AF. But because S-AF only repeats information once whereas AP-AF repeats $m$ times, S-AF actually has a higher throughput.

[Figure 3 about here.]

Figure 3 plots the throughput of S-AF and OPA AP-AF schemes. We consider a network in which all channels have equal gain, i.e., $h_{s, d}, h_{s, i}, h_{i, d} \sim \mathcal{C N}(0,1)$. As the number of relays increases, the throughput of AP-AF actually decreases due to the lower TDMA factor of $1 /(m+1)$ in (9). The figure shows that S-AF achieves much larger average throughput than AP-AF. As expected, the gains of using S-AF over 
AP-AF increases with increasing $m$.

\section{B. Outage Analysis}

In this section we compare the outage probability for the two AF schemes. We prove that S-AF scheme can also achieve full diversity order. Combined with the throughput analysis in the previous section, we can expect that S-AF has better outage probability than AP-AF in general since it has larger average throughput and the same diversity order. This is verified with simulation results in the next section.

Theorem 4: At high SNR, the outage probability of the S-AF scheme can be approximated as

$$
P_{\text {out }}^{S}=P\left[\mathcal{I}_{S}<R\right] \approx \frac{\lambda_{0} \prod_{i=1}^{m}\left(\lambda_{i}+\xi_{i}\right)}{m+1}\left(\frac{2^{2 R}-1}{\gamma}\right)^{m+1},
$$

\section{Proof: See Appendix D.}

Theorem 4 states that full diversity order of $(m+1)$ can be achieved by the S-AF scheme since as AP-AF, $P_{\text {out }}^{S}$ is proportional to $(1 / \gamma)^{m+1}$. Next, by comparing the high SNR outage probability of S-AF with the lower bound of AP-AF in Theorem 1 we arrive at the next corollary.

Corollary 1: In the high SNR regime, $P_{\text {out }}^{S}<P_{\text {out }}^{A P}$ when the target rate $R$ satisfies $R>\left(\log _{2} m\right) /(m-1)$.

Note that the condition $R>\left(\log _{2} m\right) /(m-1)$ is obtained by using the lower bound of AP-AF, and therefore is sufficient but not necessary i.e., even when the condition is not satisfied, S-AF may still have smaller outage probability than AP-AF. Furthermore, although the corollary is based on the high SNR assumption, simulation results shows that it is also valid for the medium SNR regime. The threshold $\left(\log _{2} m\right) /(m-1)$ is easily reached in practice. For instance, when $m=8$ potential relays, the required target rate is only $R>3 / 7$ bits/time slot. Therefore we can safely say that in practice S-AF provides better outage performance than AP-AF.

\section{Simulation Results}

[Figure 4 about here.]

The following figures simulate the outage probabilities for the three schemes, S-AF, AP-AF and direct transmission. In Fig. 4 the equal-gain channels as in Fig. 3 are assumed. The required outage is set to be $R=1$. The figure shows that $\mathrm{S}-\mathrm{AF}$ achieves a huge improvement in outage probability of about $4 \mathrm{~dB}$ over AP-AF, even with OPA, while both achieve full diversity order.

Figure 5 shows a more practical situation where channels are determined by the locations of nodes, as in Fig. 2. With a gain of approximately $5 \mathrm{~dB}$, the superiority of the S-AF scheme is again confirmed. 
[Figure 5 about here.]

\section{Selection AF with Power Allocation}

In the previous sections it is shown that S-AF outperforms AP-AF with optimal power allocation in terms of system throughput and outage behavior. In fact, those two techniques are not mutually exclusive. In this section, we will find the best power allocation between source and selected relay in S-AF for further improvement. Since S-AF requires complete CSI and feedback channel already, it can be combined with optimal power allocation. Thus in this section, we will focus on OPA with complete CSI.

With power allocation, the selection criterion is different: It is determined by not only the channel conditions related to the relay node, but also the source-destination direct link. Therefore we need to consider the optimal power allocation between the source and single relay first.

In a single relay network, assume the total transmission energy is $E_{T}$. The source node uses $\rho E_{T}$ for its transmission and the rest $(1-\rho) E_{T}$ is for the relay node $i$. Denote $A_{0}=a_{0} E_{T}, A_{i}=a_{i} E_{T}$ and $B_{i}=b_{i} E_{T}$, where $a_{0}, a_{i}$ and $b_{i}$ are defined in Section III-A. The received SNR (defined in Section III-C) can be rewritten as

$$
\gamma_{r}=A_{0} \rho+\frac{A_{i} B_{i} \rho(1-\rho)}{A_{i} \rho+B_{i}(1-\rho)+1} .
$$

Since maximizing instantaneous receive SNR minimizes outage probability and SER at the same time, our goal is thus to choose the optimal transmission power ratio $\rho^{o p t}$. The result is given in the following theorem.

Theorem 5: The optimal power ratio for the source node in a single relay system is

$$
\rho^{\text {opt }}=\left\{\begin{array}{ll}
1 & D_{i}<0 \\
\min \left(1, \frac{B_{i}+1}{B_{i}-A_{i}}-\frac{C_{i}}{D_{i}\left(B_{i}-A_{i}\right)}\right) & D_{i}>0
\end{array},\right.
$$

where $C_{i}=A_{i} B_{i}\left(A_{i}+1\right)\left(B_{i}+1\right)$ and $D_{i}=A_{i} B_{i}+A_{0} B_{i}-A_{0} A_{i}$. It should be noted that $A_{i}$ and $B_{i}$ are generally not equal, since they are realizations of two independent exponential distributions.

Proof: See Appendix E.

Based on Theorem 5, the OPA algorithm for S-AF can be listed as

1) For each relay node $i$, compute the optimal power ratio $\rho_{i}^{o p t}$ using (32). 
2) Compute the received SNR with optimal power ratio using node $i$ as

$$
\gamma_{r, i}^{o p t}=A_{0} \rho_{i}^{o p t}+\frac{A_{i} B_{i} \rho_{i}^{o p t}\left(1-\rho_{i}^{o p t}\right)}{A_{i} \rho_{i}^{o p t}+B_{i}\left(1-\rho_{i}^{o p t}\right)+1} .
$$

3) Choose the best node based on the received SNR using each potential relay.

4) Use the feedback channel to notify the selected relay and the source of their power level.

[Figure 6 about here.]

Figure 6 compares the outage behavior of S-AF with and without OPA. The system used in this simulation is the same as in Fig. 4. From the figure we can see that the S-AF system outage behavior is greatly improved by power allocation, especially in the high SNR regime. In this simulation, we also notice that the two S-AF schemes choose different "best" nodes with about $40 \%$ probability. This verifies that power allocation has a significant impact on the S-AF scheme.

\section{CONCLUSION}

Cooperative diversity is a powerful idea to achieve spatial diversity even when multiple antennas are unavailable at each node. Previous works have developed several schemes to realize this cooperative diversity gain, among which Amplify-and-Forward is attractive for its low complexity. The conventional $\mathrm{AF}$ (or AP-AF) scheme assumes that all the relay nodes participate in packet forwarding, and that the same power is used at all the nodes. In this paper we first consider two levels of channel information, complete CSI and channel statistics, and derive optimal power allocation among the relay nodes to minimize system outage probability with total and individual power constraints. We showed that the optimal power allocation can be obtained by an extended water-filling process. We also show that these OPA schemes also minimize SER. Another important contribution of this paper is a selection scheme, called S-AF, where only one relay node is chosen to relay the source signal. We showed that S-AF maintains full diversity order while greatly increasing the throughput, and therefore also achieves better outage behavior than AP-AF, even with OPA. Finally, OPA and S-AF may be combined for better performance.

\section{APPENDIX}

\section{A. Proof of Theorem 1}

The capacity of the AP-AF scheme is

$$
\mathcal{I}_{A P}=\frac{1}{m+1} \log _{2}\left[1+\gamma \alpha_{0}+\sum_{i=1}^{m} \frac{\gamma^{2} \alpha_{i} \beta_{i}}{\gamma \alpha_{i}+\gamma \beta_{i}+1}\right] .
$$


Thus the outage probability $P_{\text {out }}^{A P}$ is

$$
\begin{aligned}
P_{\text {out }}^{A P} & =P\left[\mathcal{I}_{A P}<R\right] \\
& =P\left[\alpha_{0}+\sum_{i=1}^{m} \frac{\gamma \alpha_{i} \beta_{i}}{\gamma \alpha_{i}+\gamma \beta_{i}+1}<\frac{2^{(m+1) R}-1}{\gamma}\right]
\end{aligned}
$$

Since

$$
\sum_{i=1}^{m} \frac{\gamma^{2} \alpha_{i} \beta_{i}}{\gamma \alpha_{i}+\gamma \beta_{i}+1}>\max _{i} \frac{\gamma^{2} \alpha_{i} \beta_{i}}{\gamma \alpha_{i}+\gamma \beta_{i}+1}
$$

an upper bound for $P_{\text {out }}^{A P}$ can be introduced as

$$
\begin{aligned}
\overline{P_{\text {out }}^{A P}} & =P\left[\frac{1}{m+1} \log _{2}\left(1+\gamma \alpha_{0}+\max _{i} \frac{\gamma^{2} \alpha_{i} \beta_{i}}{\gamma \alpha_{i}+\gamma \beta_{i}+1}\right)<R\right], \\
& =P\left[\alpha_{0}+\max _{i} \frac{\gamma \alpha_{i} \beta_{i}}{\gamma \alpha_{i}+\gamma \beta_{i}+1}<\frac{2^{(m+1) R}-1}{\gamma}\right], \\
& =P\left[\max _{i} \frac{\gamma \alpha_{i} \beta_{i}}{\gamma \alpha_{i}+\gamma \beta_{i}+1}<\delta-\alpha_{0}\right],
\end{aligned}
$$

where $\delta=\frac{2^{(m+1) R}-1}{\gamma}$. Since $\alpha_{0}$ is exponentially distributed variable with parameter $\lambda_{0}$, we have

$$
\begin{gathered}
\overline{P_{\text {out }}^{A P}}=\int_{0}^{\delta} P\left[\max _{i} \frac{\gamma \alpha_{i} \beta_{i}}{\gamma \alpha_{i}+\gamma \beta_{i}+1}<\delta-x\right] \lambda_{0} e^{-\lambda_{0} x} d x \\
=\int_{0}^{1} P\left[\max _{i} \frac{\gamma \alpha_{i} \beta_{i}}{\gamma \alpha_{i}+\gamma \beta_{i}+1}<\delta x^{\prime}\right] \delta \lambda_{0} e^{-\lambda_{0} \delta\left(1-x^{\prime}\right)} d x^{\prime} \\
=\int_{0}^{1}\left(\prod_{i=1}^{m} P\left[\frac{\gamma \alpha_{i} \beta_{i}}{\gamma \alpha_{i}+\gamma \beta_{i}+1}<\delta x^{\prime}\right]\right) \delta \lambda_{0} e^{-\lambda_{0} \delta\left(1-x^{\prime}\right)} d x^{\prime} \\
=\delta^{m+1} \lambda_{0} \int_{0}^{1}\left(\prod_{i=1}^{m} \frac{P\left[\frac{\gamma \alpha_{i} \beta_{i}}{\gamma \alpha_{i}+\gamma \beta_{i}+1}<\delta x^{\prime}\right]}{\delta x^{\prime}}\right)\left(x^{\prime}\right)^{m} e^{-\lambda_{0} \delta\left(1-x^{\prime}\right)} d x^{\prime}
\end{gathered}
$$

using $\left(x^{\prime}=1-x / \delta\right)$ Note that $\delta$ is a function of transmit SNR $\gamma$, and $\delta \rightarrow 0$ when $\gamma \rightarrow \infty$. Thus

$$
\lim _{\gamma \rightarrow \infty} e^{-\lambda_{0} \delta\left(1-x^{\prime}\right)}=1
$$

By Lemma 1 in Appendix 1 of [2], we have

$$
\lim _{\gamma \rightarrow \infty} \frac{P\left[\frac{\gamma \alpha_{i} \beta_{i}}{\gamma \alpha_{i}+\gamma \beta_{i}+1}<\delta x^{\prime}\right]}{\delta x^{\prime}}=\lambda_{i}+\xi_{i} .
$$


Substituting (38) and (39) into (37), and using the dominated convergence theorem [18] we have

$$
\begin{aligned}
\lim _{\gamma \rightarrow \infty} \frac{\overline{P_{\text {out }}^{A P}}}{\delta^{m+1}} & =\lambda_{0} \int_{0}^{1}\left(\prod_{i=1}^{m}\left(\lambda_{i}+\xi_{i}\right)\right)\left(x^{\prime}\right)^{m} d x^{\prime} \\
& =\frac{\lambda_{0} \prod_{i=1}^{m}\left(\lambda_{i}+\xi_{i}\right)}{m+1} .
\end{aligned}
$$

Therefore, at sufficiently high SNR, $P_{\text {out }}^{A P}$ can be upper bounded by

$$
P_{\text {out }}^{A P}<\frac{\lambda_{0} \prod_{i=1}^{m}\left(\lambda_{i}+\xi_{i}\right)}{m+1} \delta^{m+1}
$$

For the lower bound, we use the inequality

$$
\sum_{i=1}^{m} \frac{\gamma^{2} \alpha_{i} \beta_{i}}{\gamma \alpha_{i}+\gamma \beta_{i}+1}<m \max _{i} \frac{\gamma^{2} \alpha_{i} \beta_{i}}{\gamma \alpha_{i}+\gamma \beta_{i}+1},
$$

therefore

$$
\begin{aligned}
& P_{\text {out }}^{A P} \quad>\quad \underline{P_{\text {out }}^{A P}} \\
& =P\left[\alpha_{0}+m \max _{i} \frac{\gamma \alpha_{i} \beta_{i}}{\gamma \alpha_{i}+\gamma \beta_{i}+1}<\frac{2^{(m+1) R}-1}{\gamma}\right] \\
& =P\left[\max _{i} \frac{\gamma \alpha_{i} \beta_{i}}{\gamma \alpha_{i}+\gamma \beta_{i}+1}<\frac{\frac{2^{(m+1) R}-1}{\gamma}-\alpha_{0}}{m}\right] \\
& =\int_{0}^{\delta} P\left[\max _{i} \frac{\gamma \alpha_{i} \beta_{i}}{\gamma \alpha_{i}+\gamma \beta_{i}+1}<\frac{\frac{2^{(m+1) R}-1}{\gamma}-x}{m}\right] \lambda_{0} e^{-\lambda_{0} x} d x \\
& =\left(\frac{2^{(m+1) R}-1}{\gamma}\right)^{m+1} \frac{\lambda_{0}}{m^{m}} \int_{0}^{1}\left(\prod_{i=1}^{m} \frac{P\left[\frac{\gamma \alpha_{i} \beta_{i}}{\gamma \alpha_{i}+\gamma \beta_{i}+1}<\frac{2^{(m+1) R}-1}{m \gamma} x^{\prime}\right]}{\frac{2^{(m+1) R}-1}{m \gamma} x^{\prime}}\right)\left(x^{\prime}\right)^{m} e^{-\lambda_{0} \frac{2^{(m+1) R}-1}{\gamma}\left(1-x^{\prime}\right)} d x^{\prime} \\
& \underset{\sim \operatorname{highSNR}}{\approx} \frac{\lambda_{0} \prod_{i=1}^{m}\left(\lambda_{i}+\xi_{i}\right)}{(m+1) m^{m}}\left(\frac{2^{(m+1) R}-1}{\gamma}\right)^{m+1},
\end{aligned}
$$

where the high SNR approximation can be easily derived using (38) and (39).

Combining (41) and (43) finishes the proof of Theorem 1.

\section{B. Optimal Power Allocation on AP-AF}

The Lagrangian of optimization problem (15) is

$$
\mathcal{L}\left(E_{i}, \lambda\right)=\sum_{i=1}^{m} \frac{E_{s}^{2} a_{i}^{2}+E_{s} a_{i}}{E_{s} a_{i}+E_{i} b_{i}+1}+\frac{1}{\lambda^{2}}\left(\sum_{i=1}^{m} E_{i}\right)
$$


where Lagrange multiplier is set to be $1 / \lambda^{2}$ to simplify the final result.

Setting the derivative of the Lagrangian w.r.t. $E_{i}$ to zero we get

$$
\begin{aligned}
& \frac{-b_{i}\left(E_{s}^{2} a_{i}^{2}+E_{s} a_{i}\right)}{\left(E_{s} a_{i}+E_{i} b_{i}+1\right)^{2}}+\frac{1}{\lambda^{2}}=0 \\
& E_{i}=\sqrt{\frac{E_{s}^{2} a_{i}^{2}+E_{s} a_{i}}{b_{i}}} \lambda-\frac{E_{s} a_{i}+1}{b_{i}} .
\end{aligned}
$$

Since the power allocated to each relay node can only be non-negative, we can use the KKT conditions [15] to verify that optimal power allocation is

$$
E_{i}=\left(\sqrt{\frac{E_{s}^{2} a_{i}^{2}+E_{s} a_{i}}{b_{i}} \lambda}-\frac{E_{s} a_{i}+1}{b_{i}}\right)^{+}
$$

where $\lambda$ is set to satisfy the total power constraint.

\section{Proof of Theorem 3}

The Lagrangian of optimization problem (22) without individual power constraints can be written as

$$
\mathcal{L}\left(E_{i}, \lambda\right)=\sum_{i=1}^{m} \log \left(\frac{\eta_{s, i}}{E_{s} \nu_{s, i}}+\frac{\eta_{i, d}}{E_{i} \nu_{i, d}}\right)+\frac{1}{\lambda}\left(\sum_{i=1}^{m} E_{i}\right) .
$$

Note we take logarithm of the objective function to simplify the derivation. Here we choose the Lagrange Multiplier as $1 / \lambda$ also for simplicity.

Setting the derivative of the Lagrangian w.r.t. $E_{i}$ to zero we get

$$
\frac{-\frac{\eta_{i, d}}{E_{i}^{2} \nu_{i, d}}}{\frac{\eta_{s, i}}{E_{s} \nu_{s, i}}+\frac{\eta_{i, d}}{E_{i} \nu_{i, d}}}+\frac{1}{\lambda}=0
$$

or,

$$
c_{i} E_{i}^{2}+E_{s} E_{i}-\lambda E_{s}=0
$$

where $c_{i}=\frac{\nu_{i, d} \eta_{s, i}}{\nu_{s, i} \eta_{i, d}}$.

Solving (49) we get two solutions. Since $\frac{-\sqrt{4 c_{i} E_{s} \lambda+E_{s}^{2}}-E_{s}}{2 c_{i}}$ is always negative, it is a invalid solution. Thus we have

$$
E_{i}=\frac{\sqrt{4 c_{i} E_{s} \lambda+E_{s}^{2}}-E_{s}}{2 c_{i}}
$$

For individual power constraints, we can use the KKT conditions to verify that optimal power allocation 
is on the boundary. Therefore, the optimal power is

$$
E_{i}=\left(\frac{\sqrt{4 c_{i} E_{s} \lambda+E_{s}^{2}}-E_{s}}{2 c_{i}}\right)_{0}^{E_{i}^{\max }} .
$$

\section{Proof of Theorem 4}

Based on the capacity formula (29), the outage probability of S-AF scheme can be written as

$$
\begin{aligned}
P_{\text {out }}^{S} & =P\left[\frac{1}{2} \log _{2}\left(1+\gamma \alpha_{0}+\max _{i} \frac{\gamma^{2} \alpha_{i} \beta_{i}}{\gamma \alpha_{i}+\gamma \beta_{i}+1}\right)<R\right] \\
& =P\left[\alpha_{0}+\max _{i} \frac{\gamma \alpha_{i} \beta_{i}}{\gamma \alpha_{i}+\gamma \beta_{i}+1}<\frac{2^{2 R}-1}{\gamma}\right], \\
& =P\left[\alpha_{0}+\max _{i} \frac{\gamma \alpha_{i} \beta_{i}}{\gamma \alpha_{i}+\gamma \beta_{i}+1}<\delta\right]
\end{aligned}
$$

where $\delta=\frac{2^{2 R}-1}{\gamma}$, which is different from the one in (36).

Using (40), at high SNR, $P_{\text {out }}^{S}$ can be approximated as

$$
P_{\text {out }}^{S} \approx \frac{\lambda_{0} \prod_{i=1}^{m}\left(\lambda_{i}+\xi_{i}\right)}{m+1} \delta^{m+1} .
$$

Theorem 4 is proved.

\section{E. Proof of Theorem 5}

The optimization problem is

$$
\rho^{o p t}=\arg \min _{0 \leq \rho \leq 1} A_{0} \rho+\frac{A_{i} B_{i} \rho(1-\rho)}{A_{i} \rho+B_{i}(1-\rho)+1} .
$$

First ignore the constraint, take the derivative of the objective function and set it to zero we have

$$
D_{i}\left(B_{i}-A_{i}\right) \rho^{2}-2 D_{i}\left(B_{i}+1\right) \rho+\left(A_{0} B_{i}+A_{i} B_{i}+A_{0}\right)\left(B_{i}+1\right)=0,
$$

where $D_{i}$ is as defined in the theorem.

The above equation has no real number solution when $D_{i}<0$. In this case the derivative of the objective function never changes sign, therefore it is a monotone function. Due to the fact that

$$
\gamma_{r}(\rho=1)=A_{0}>0=\gamma_{r}(\rho=0)
$$


it must be a monotonically increasing function. Therefore $\rho^{\text {opt }}=1$.

If $D_{i}>0$, the equation has two real solutions. It is easy to show that one solution is always outside the possible regime $(0,1)$. Ignoring this solution, we get

$$
\rho^{o p t}=\frac{B_{i}+1}{B_{i}-A_{i}}-\frac{C_{i}}{D_{i}\left(B_{i}-A_{i}\right)}
$$

If $\rho^{\text {opt }}$ is outside $(0,1)$, Karush-Kuhn-Tucker condition guarantees that the optimal solution lies on the boundary. In this case $\rho^{\text {opt }}=1$.

Theorem 5 is proved. 


\section{REFERENCES}

[1] J. N. Laneman and G. W. Wornell, "Distributed space-time-coded protocols for exploiting cooperative diversity in wireless networks," IEEE Transactions on Information Theory, vol. 49, pp. 2415-2425, November 2003.

[2] J. N. Laneman, D. N. C. Tse, and G. W. Wornell, "Cooperative diversity in wireless networks: Efficient protocols and outage behavior," IEEE Transactions on Information Theory, vol. 50, pp. 3062-3080, December 2004.

[3] H. El Gamal and D. Aktas, "Distributed space-time filtering for cooperative wireless networks," Proceedings of IEEE Globecom, pp. 1826-1830, 2003.

[4] Y. Liang and V. V. Veeravalli, "Resource allocation for wireless relay channels," Proceedings of the 38th Asilomar Conference on Signals, Systems, and Computers, pp. 1902-1906, November 2004.

[5] S. Serbetli and A. Yener, "Power allocation strategies for relay assisted communications," WICAT Workshop on Cooperative Communications, October 2005.

[6] Y. Yao, X. Cai, and G. B. Giannakis, “On energy efficiency and optimum resource allocation of relay transmissions,” IEEE Transactions on Wireless Communications, vol. 4, pp. 2917-2927, November 2005.

[7] A. Host-Madsen and J. Zhang, "Capacity bounds and power allocation in wireless relay channel," IEEE Transactions on Information Theory, vol. 51, pp. 2020-2040, June 2005.

[8] X. Deng and A. M. Haimovich, "Power allocation for cooperative relaying in wireless networks," IEEE Communications Letters, vol. 9, pp. 994-996, November 2005.

[9] D. R. Brown III, "Resource allocation for cooperative transmission in wireless networks with orthogonal users," Proceedings of the 38th Asilomar Conference on Signals, Systems, and Computers, November 2004.

[10] M. O. Hasna and M. Alouini, "Optimal power allocation for relayed transmissions over Rayleigh fading channels," IEEE Transactions on Wireless Communications, vol. 3, pp. 1999-2004, November 2004.

[11] I. Hammerstroem, M. Kuhn, and A. Wittneben, "Impact of relay gain allocation on the performance of cooperative diversity networks," Proceedings of IEEE Vehicular Technology Conference, VTC Fall, September 2004.

[12] A. Bletsas, A. Lippman, and D. Reed, "A simple distributed method for relay selection in cooperative diversity wireless networks, based on reciprocity and channel measurements," Proceedings of IEEE 61st Vehicular Technology Conference, May 2005.

[13] E. Beres and R. Adve, “On selection cooperation in distributed networks," Proceedings of IEEE 40th Conference on Information Science and Systems, March 2006.

[14] R. U. Nabar, H. Bolcskei, and F. W. Kneubuhler, "Fading relay channels: Performance limits and space-time signal design," IEEE Journal on Selected Areas in Communications, vol. 22, pp. 1099-1109, August 2004.

[15] D. Luenberger, Linear and Nonlinear Programming: 2nd Edition. Addison-Wesley, 1984.

[16] L. Zheng and D. N. Tse, "Diversity and multiplexing: A fundamental tradeoff in multiple-antenna channels," IEEE Transactions on Information Theory, vol. 49, pp. 1073-1096, May 2003.

[17] A. Ribeiro, X. Cai, and G. Giannakis, "Symbol error probabilities for general cooerative links," IEEE Transactions on Wireless Communications, vol. 4, pp. 1264-1273, May 2005.

[18] A. Browder, Mathematical Analysis: An Introduction. New York: Springer-Verlag, 1996. 


\section{LIST OF FIGURES}

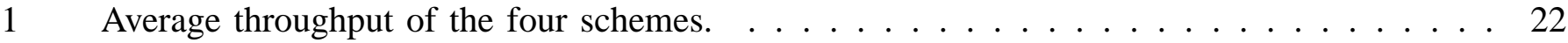

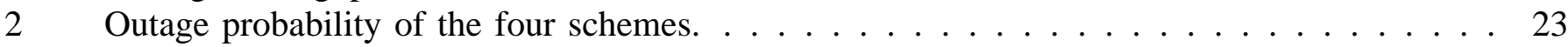

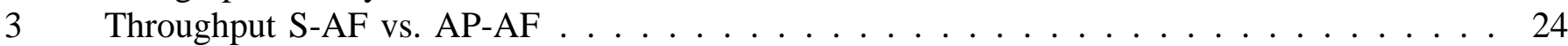

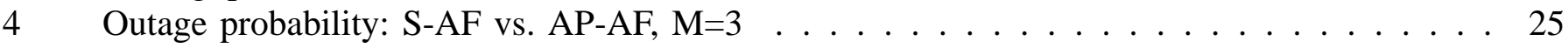

5 Outage probabilities for three schemes, location determines channel, $\mathrm{M}=3 \ldots \ldots \ldots \ldots$

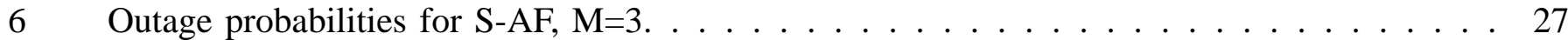




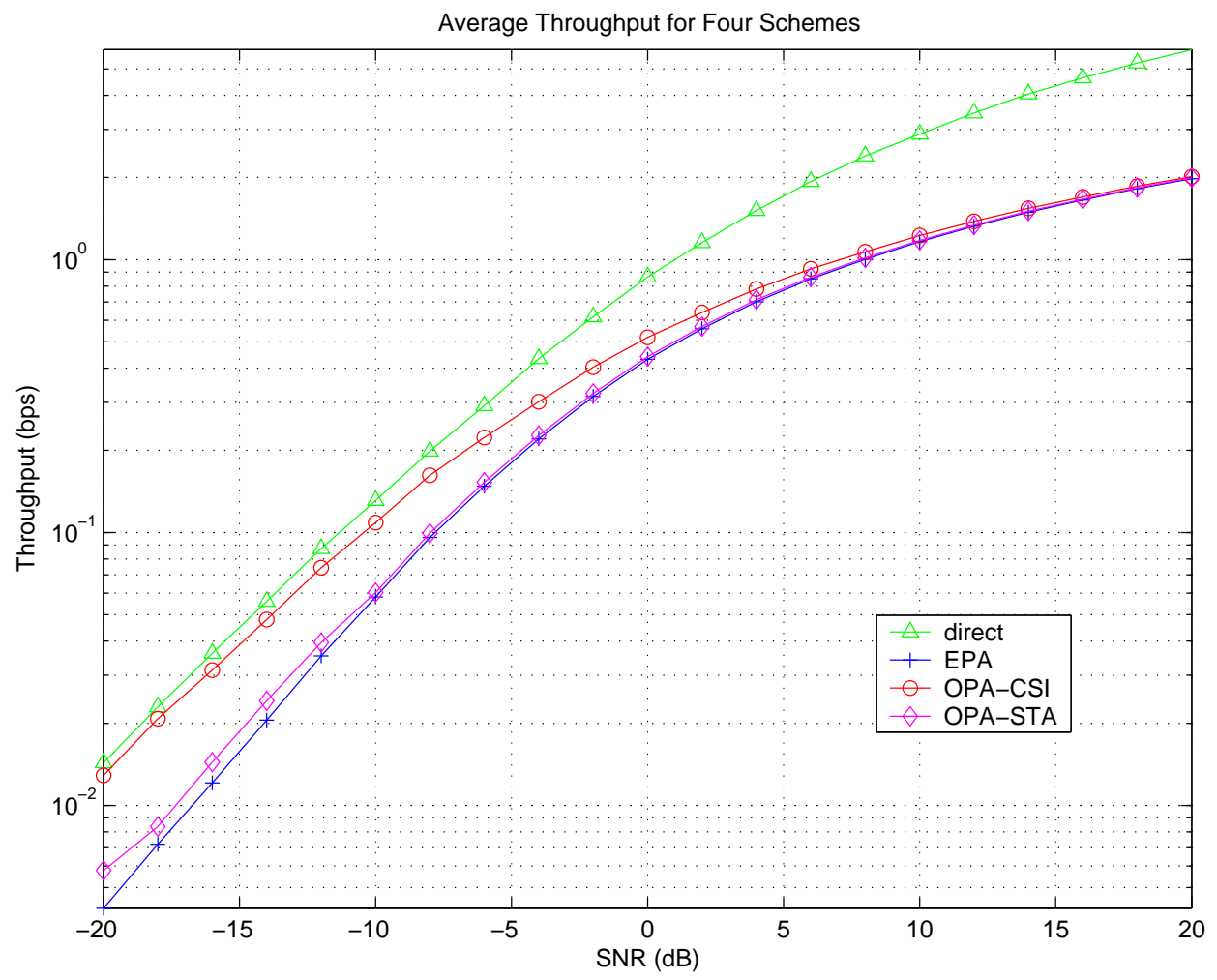

Fig. 1. Average throughput of the four schemes. 


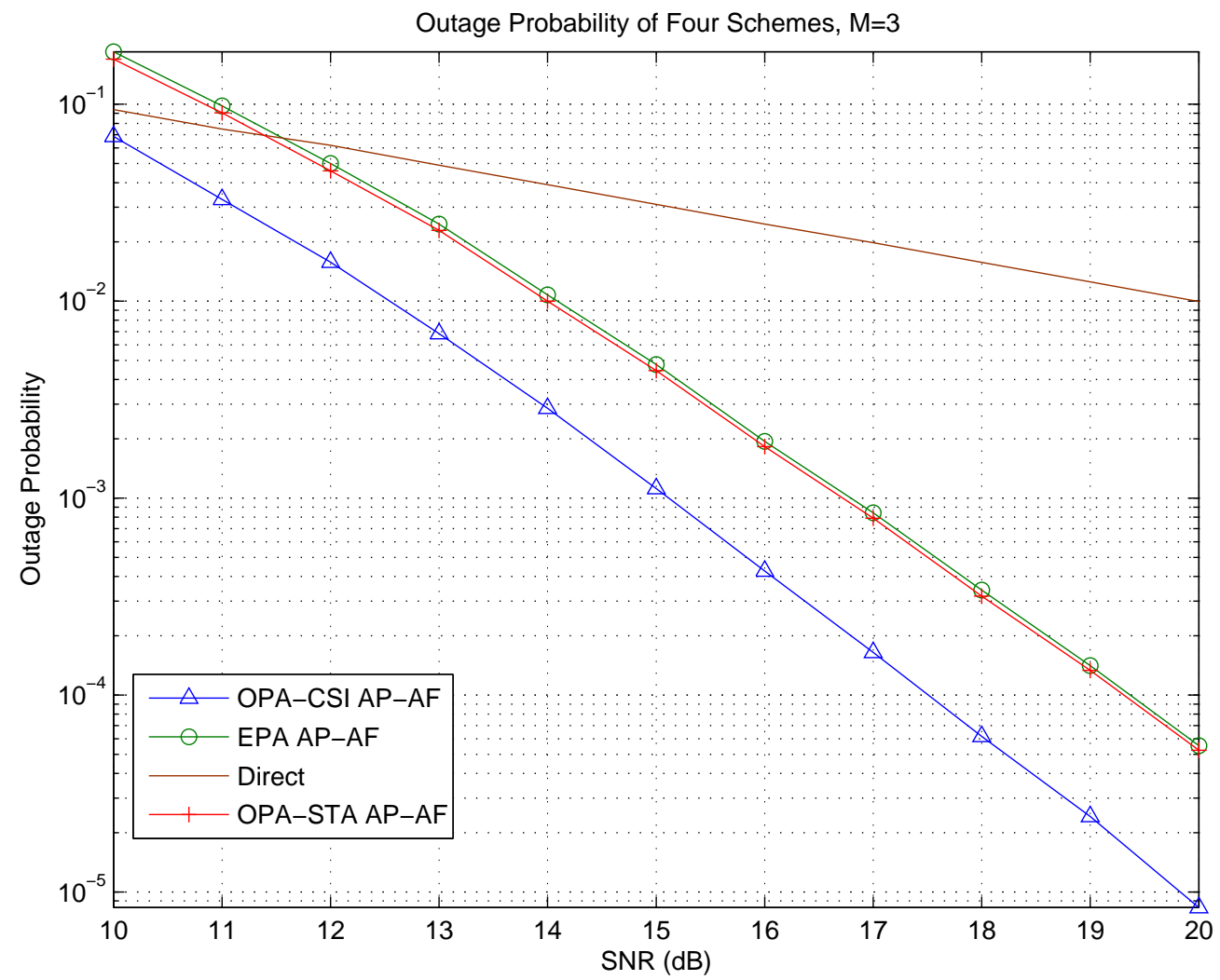

Fig. 2. Outage probability of the four schemes. 


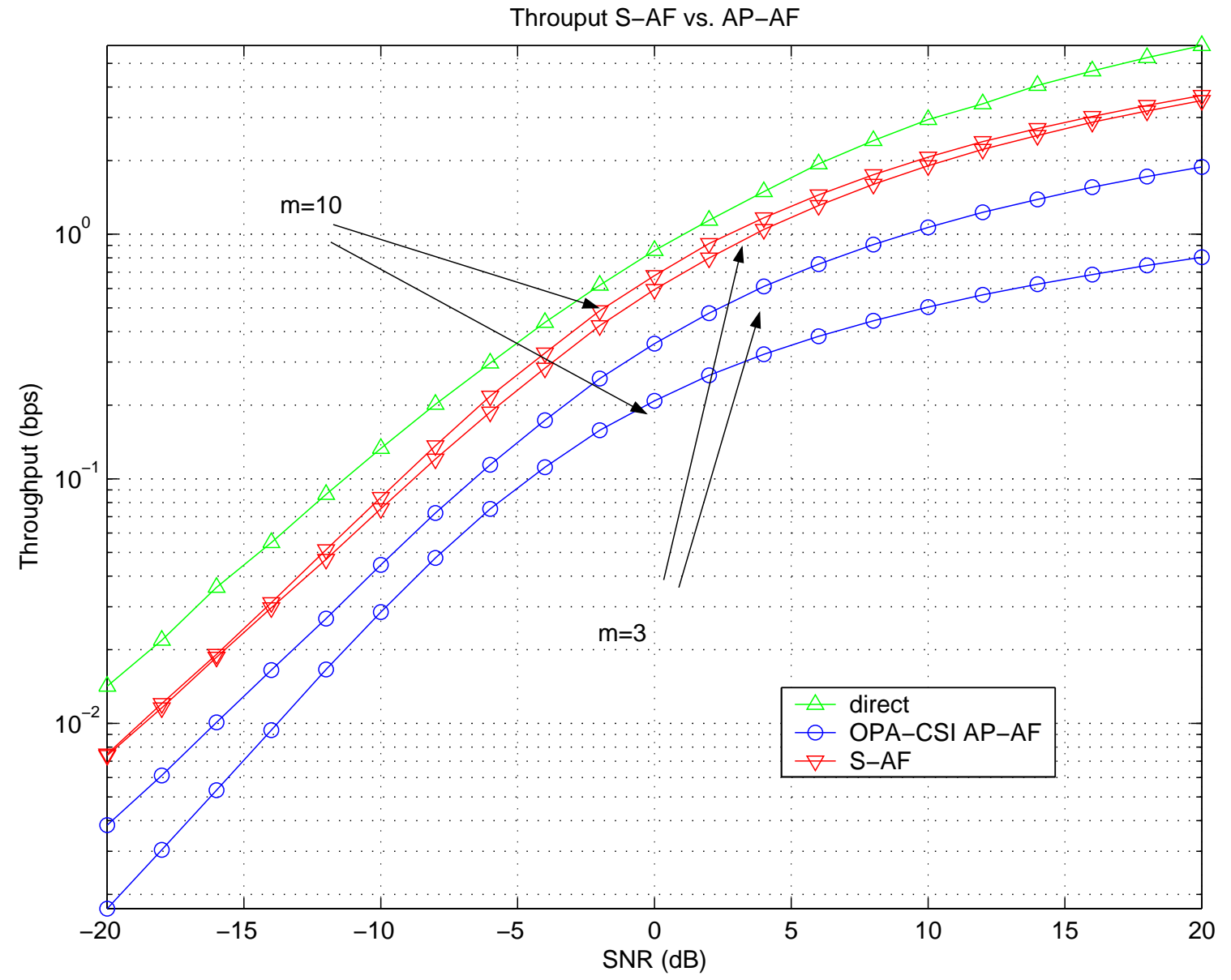

Fig. 3. Throughput S-AF vs. AP-AF 


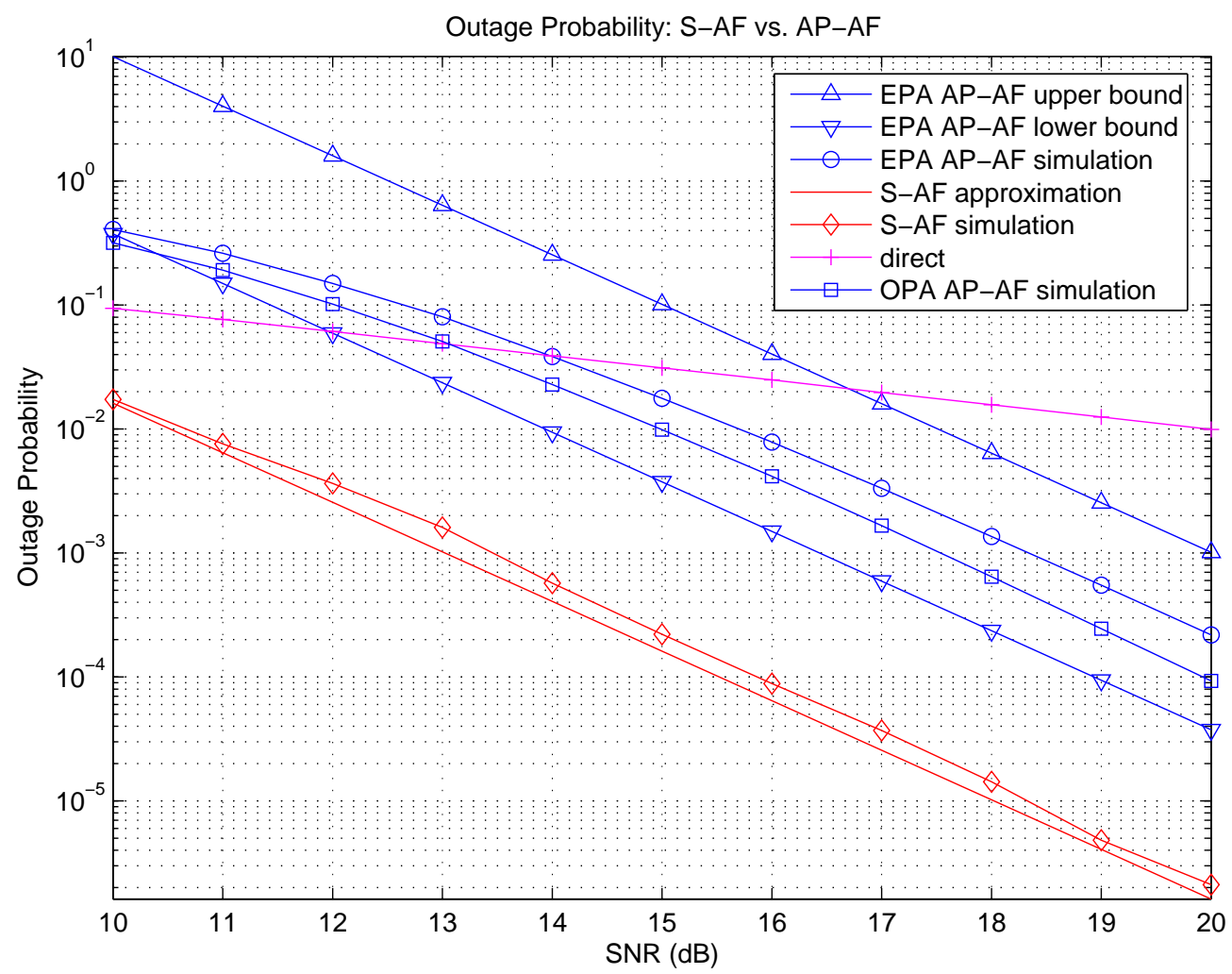

Fig. 4. Outage probability: S-AF vs. AP-AF, M=3 


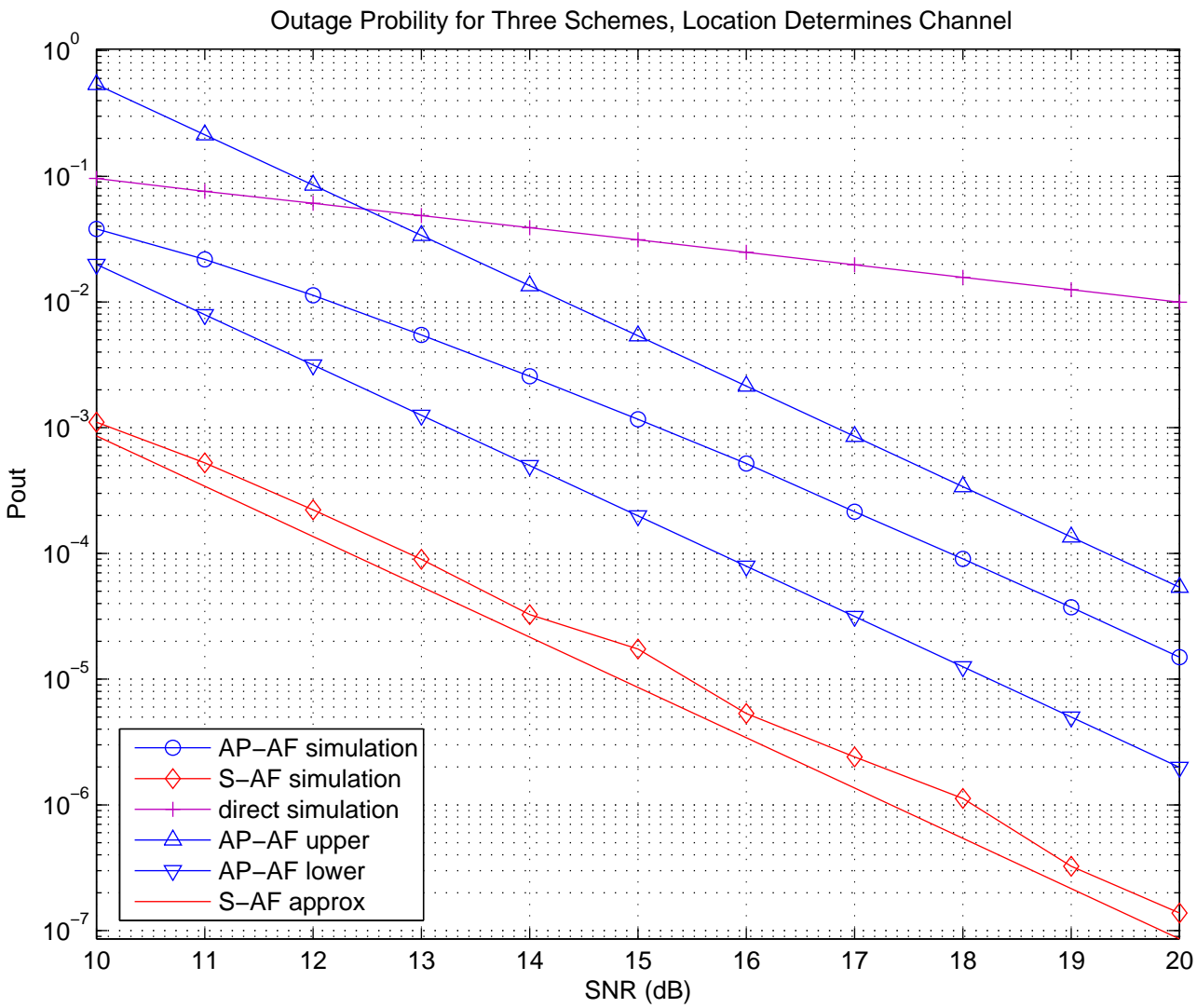

Fig. 5. Outage probabilities for three schemes, location determines channel, $M=3$. 


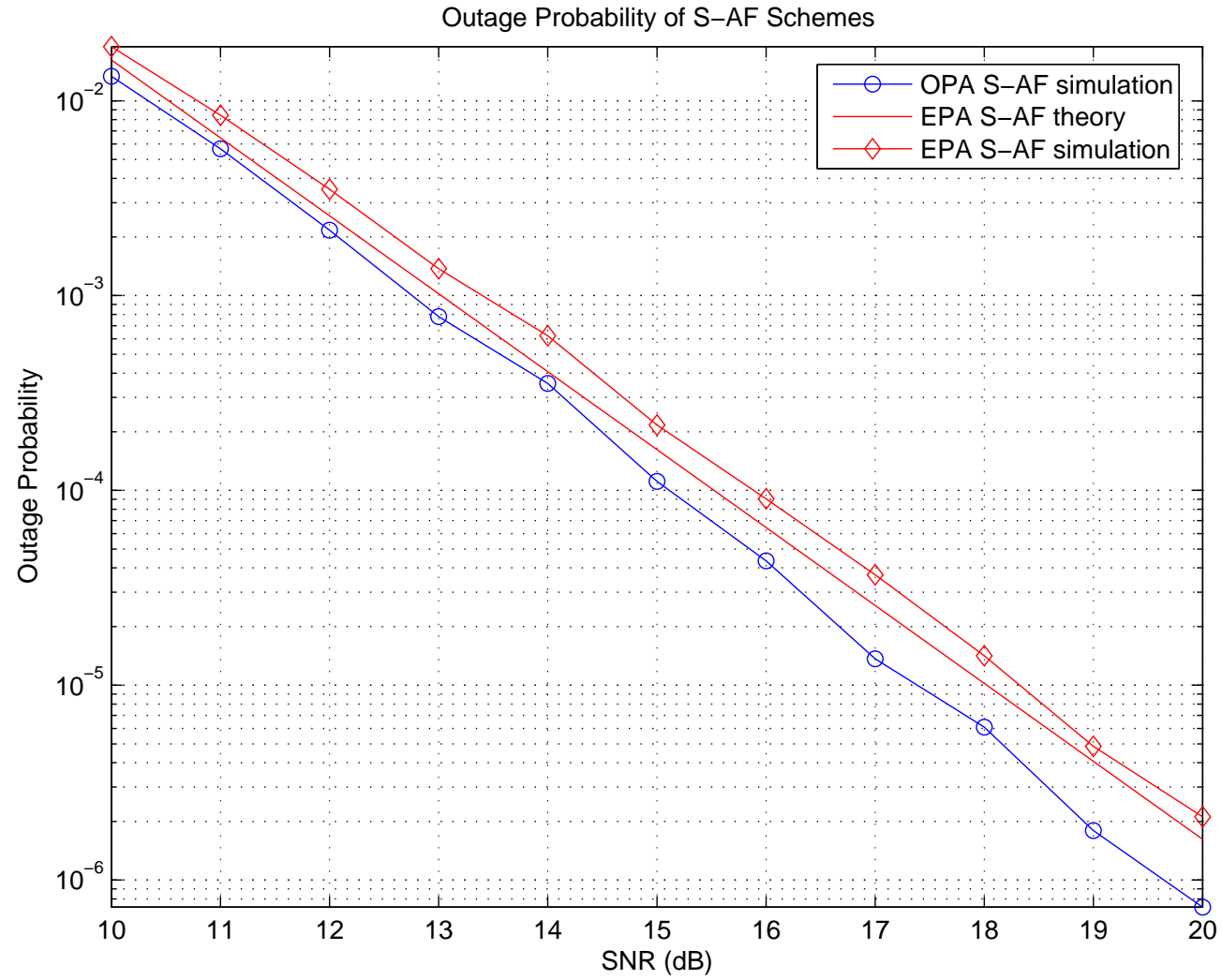

Fig. 6. Outage probabilities for S-AF, M=3. 